\title{
ON A UNIQUENESS PROBLEM \\ IN THE THEORY OF LINEAR INTEGRAL EQUATIONS
}

\author{
ROBERT R. STEVENS
}

ABSTRACT. The primary purpose of this paper is to give sufficient conditions for a function $G$ which ensure that if $\int_{0}^{1} f(x t) G(t) d t=0$ a.e. in $(0,1)$ then the function $f$ is zero almost everywhere in $(0,1)$. Several applications are given.

1. Introduction. A fundamental result in the theory of real functions is the following: if $f(x)$ is Lebesgue integrable on an interval $(a, b)$ and if $\int_{a}^{x} f(t) d t=0$ for all $x \in(a, b)$, then $f$ is equivalent to zero $(f=0$ a.e. $)$ in $(a, b)$. (In this paper, all integrals are to be interpreted as Lebesgue integrals. The terms "almost everywhere", "measurable", etc. refer to Lebesgue measure in the interval $(0,1)$.) This result may be rephrased as

Theorem. If $f \in L^{1}(0,1)$ and $\int_{0}^{1} f(x t) d t=0$ for all $x \in(0,1]$, then $f$ is equivalent to zero in $(0,1)$.

Since proofs [2], [3] of this have relied heavily upon the translation invariance of the Lebesgue measure $d t$, it is of some interest to consider the following (uniqueness) problem:

What conditions on the function $G(t)$ will ensure that $f$ is equivalent to zero (in $(0,1))$ if

(i) $f$ belongs to a certain function class (e.g., $f$ analytic, $f \in C^{\infty}[0,1]$, $f \in L^{p}[0,1]$, etc.), and

(ii) $\int_{0}^{1} f(x t) G(t) d t=0$ for all $x \in(0,1]$.

One might also formulate the problem in terms of a measure rather than a density function $G$, but we shall not do this here.

Examples of this general problem occur in several contexts: the uniqueness of the solution of Schlömilch's integral equation in the theory of Bessel function expansions [6], [7], Urabe's [5] characterization of those functions $g(x)$ for which all solutions of $x^{\prime \prime}+g(x)=0$ have a common period, Mellin transform solutions of integral equations [1], etc.

Presented to the Society, January 18, 1974 under the title Uniqueness theorems for the generalized Schlömilch equation; received by the editors February 4, 1974.

AMS (MOS) subject classifications (1970). Primary 45A05; Secondary 30A08, 34C $25,39 A 15$. 
For $f$ analytic, the problem is a fairly easy one [5], but the $C^{\infty}$ case appears to be more difficult. Theorem 4 below concerns this case.

More importantly, the results here pertain to the general $L^{1}$ case. Examples showing that the results are, in a sense, best possible are presented. Finally, an application is given which shows that the incomplete gamma function $P(z)=\int_{0}^{1} t^{z-1} e^{-t} d t$ is zero-free in the right half-plane $\operatorname{Re} z>0$.

2. The $L^{1}$ case.

Theorem 1. Let $\int_{0}^{1} f(x t) G(t) d t=q(x) f(x)$ for almost all $x \in(0,1)$ where (i) $f \in L^{1}(0,1)$,

(ii) $G$ is positive and nondecreasing on $(a, 1]$ and $G=0$ on $[0, a)$ for some $a \in[0,1)$,

(iii) $q$ is a measurable and nonpositive $(q \leqq 0)$ function on $(0,1)$. Then $f=0$ almost everywhere in $(0,1)$.

For the proof of this theorem, we require the following lemma.

Lemma 1. Let $G$ be a positive and nondecreasing function on an open interval $(a, b)$ and let $f$ and $f \cdot G(f(x) G(x))$ belong to $L^{1}(a, b)$. Then for any $\epsilon>0$ there exists a positive and absolutely continuous function $H$ on $[a, b]$ satisfying

(i) $H^{\prime} \geq 0$ almost everywhere in $[a, b]$,

(ii) $\int_{a}^{b} \bar{f}(x)[H(x)-G(x)] d x<H(a) \epsilon$,

(iii) $H(a) \geqq G\left(a^{+}\right)$,

(iv) $H(b)-H(a)=G\left(b^{-}\right)-G\left(a^{+}\right)$, in case that $G$ is also bounded in $(a, b)$.

The proof of Lemma 1, involving standard arguments concerning partition of the range of $G$, is omitted. (Indeed, $H$ may be taken as a piecewise linear function.)

Proof of Theorem 1. (We shall give the proof for the case $a=0$, since . the proof for the general case is essentially the same.) We observe first that the integral $\int_{0}^{1} f(x t) G(t) d t$ is a continuous function for $x \in(0,1]$, since this may be written as

$$
x^{-1} \int_{0}^{x} f(t) G(t / x) d t
$$

and since the integral $\int_{0}^{x} f(t) G(t / x) d t$ is continuous at each point $x$ of $[0,1]$. (This follows by a straightforward application of Lebesgue's dominated convergence theorem, using the fact that the nondecreasing function $G$ is continuous almost everywhere in $[0,1]$.)

Let $F(x) \equiv \int_{0}^{x} f(t) d t$. We show first that $F \leqq 0$ for all $x \in[0,1]$. 
Assume the contrary and let $\epsilon$ be an arbitrary positive number. By the lemma, there exists a positive and absolutely continuous $H$ on $[0,1]$ such that $H^{\prime} \geqq 0$ almost everywhere in $[0,1]$ and $P\left(x_{1}\right)<H(0) \epsilon$, where

$$
P(x) \equiv \int_{0}^{1} f(x t)[H(t)-G(t)] d t
$$

and $x_{1} \in(0,1]$ is such that $F\left(x_{1}\right)=\max \{F(x) \mid 0 \leqq x \leqq 1\}$. Then

$$
\int_{0}^{1} f(x t) H(t) d t=P(x)+q(x) f(x)
$$

a. e. in $(0,1)$ and an integration by parts yields

$$
\int_{0}^{1}[F(x t)-F(x)] H^{\prime}(t) d t=H(0) F(x)-x[P(x)+q(x) f(x)] .
$$

Thus

(2.1) $0 \geqq \int_{0}^{1}\left[F\left(x_{1} t\right)-F\left(x_{1}\right)\right] H^{\prime}(t) d t=H(0) F\left(x_{1}\right)-x_{1}\left[P\left(x_{1}\right)+q\left(x_{1}\right) f\left(x_{1}\right)\right]$.

Note here that the integral equation implies that the product $q(x) f(x)$ is (equivalent to) a continuous function on $(0,1]$. Also, since $F\left(x_{1}\right)$ is the (positive) maximum value of $F, f$ must assume nonnegative values in any left neighborhood $\left(x_{1}-\delta, x_{1}\right)$ of $x_{1}$. Thus $q\left(x_{1}\right) f\left(x_{1}\right) \leqq 0$. If follows from (2.1) that

$$
0 \geqq H(0) F\left(x_{1}\right)-x_{1} P\left(x_{1}\right) \geqq H(0) F\left(x_{1}\right)-H(0)_{\epsilon}
$$

and $F\left(x_{1}\right) \leqq \epsilon$. Since $\epsilon$ is an arbitrary positive number, $F\left(x_{1}\right) \leqq 0$. This contradiction of our assumption implies that $F(x) \leqq 0$ for all $x \in[0,1]$. Repetition of the argument with $-f$ replacing $f$ yields $-F(x) \leqq 0$ for all $x \in[0,1]$. Thus, $F(x) \equiv 0$ and $f=0$ almost everywhere in $(0, \overline{1})$.

It is of some importance to obtain a theorem, similar to Theorem 1, which does not require boundedness of the function $G$. For this, a straightforward modification of the proof of Theorem 1 yields

Theorem 2. Let $\int_{0}^{1} f(x t) G(t) d t=q(x) f(x)$ for almost all $x \in(0,1)$, where

(i) $f \in L^{1}(0,1)$ and $f$ is essentially bounded in a neighborhood $(b, 1]$ of $x=1$,

(ii) $G \in L^{1}(0,1)$ and $G$ is positive and nondecreasing on $(a, 1), G=0$ on $[0, a)$ for some $a \in[0,1)$, and

(iii) $q(x)$ is a measurable and nonpositive function on $(0,1)$.

Then $f=0$ almost everywhere in $(0,1)$.

It should be noted here that the hypothesis that $f$ be essentially bounded 
in a neighborhood of $x=1$ is automatically met if, in addition, the function $q$ is bounded away from zero in a neighborhood of $x=1$.

Corollary 1. Let $\int_{0}^{1} f(x t) G(t) d t=0$ for almost all $x \in(0,1)$, where

(i) $f \in L^{1}(0,1)$,

(ii) $G$ is absolutely continuous on $[0,1], G^{\prime}<0$ and $G^{\prime}$ is nonincreasing on $(0,1)$,

(iii) $G(1) \geqq 0$.

Then $f=0$ almost everywhere in $(0,1)$.

Proof. Let $g=-G^{\prime}$ and $F(x)=\int_{0}^{x} f(t) d t$. Then $g$ is positive and nondecreasing on $(0,1)$ and $g \in L^{1}(0,1)$. $\left(\int_{0}^{1} g(t) d t=G(0)-G(1)\right.$.) An integration by parts gives

$$
\int_{0}^{1} F(x t) g(t) d t=-G(1) F(x)+x \int_{0}^{1} f(x t) G(t) d t=-G(1) F(x)
$$

for all $x$ in $[0,1]$. Finally, application of Theorem 2 to the integral equation

$$
\int_{0}^{1} F(x t) g(t) d t=-G(1) F(x)
$$

yields $F \equiv 0$ for all $x \in[0,1]$, and $f=0$ almost everywhere in $(0,1)$.

If the function $G$ is strictly positive, the monotonicity condition of Theorem 1 may be considerably relaxed, as the next result shows.

Theorem 3. Let $\int_{0}^{1} f(x t) G(t) d t=q(x) f(x)$ for almost all $x \in(0,1)$, where

(i) $f \in L^{1}(0,1)$,

(ii) $G$ is of bounded variation on $[0,1]$ and the negative variation of $G$ on $[0,1]$ is strictly less than $1 / 2 G(0+)$,

(iii) $q$ is a measurable and nonpositive function on $(0,1)$.

Then $f=0$ almost everywhere in $(0,1)$.

Proof. (For the standard results concerning positive and negative variation of functions of bounded variation, we refer to [3, p. 99].) Let $F(x)=$ $\int_{0}^{x} f(t) d t$ and suppose to the contrary that $f$ is not equivalent to zero on $(0,1)$. We shall assume, without loss of generality, that $G$ is continuous at $x=0$ and $x=1$ and note that there exists $p<1 / 2$ such that the negative variation of $G$ on $[0,1]$ is strictly less than $p G(0)$. Let

$$
F\left(x_{1}\right)=\max \{F(x) \mid 0 \leqq x \leqq 1\}, \quad F\left(x_{2}\right)=\min \{F(x) \mid 0 \leqq x \leqq 1\},
$$

and $T=F\left(x_{1}\right)-F\left(x_{2}\right)$. We may assume (replacing $f$ by $-f$ if necessary) that

$$
2 F\left(x_{1}\right) \geqq T
$$


Now let $\epsilon>0$ be an arbitrary positive number. Write $G(x)=P(x)+G(0)-$ $N(x)$, where $P(x)$ is the positive and $N(x)$ is the negative variation of $G$ on $[0, x]$, and let $G_{1}(x)=P(x)+G(0)$ and $G_{2}(x)=N(x)$, so that $G(x)$ is represented as the difference $G(x)=G_{1}(x)-G_{2}(x)$ of two nonnegative nondecreasing functions. By our assumption, $G_{2}(1)=N(1)<p G(0)$. As in I.emma 1 , there exists a positive absolutely continuous function $H_{1}$, with $H_{1} \geqq 0$ on $[0,1]$, such that

$$
\int_{0}^{1} f\left(x_{1} t\right)\left[H_{1}(t)-G_{1}(t)\right] d t<H_{1}(0)_{\epsilon} / 2
$$

and an absolutely continuous function $H_{2}$, with $H_{2}^{\prime} \geqq 0$ on $[0,1]$ and $H_{2}(0)=0$ and $H_{2}(1)=G_{2}(1)$, such that

$$
\int_{0}^{1} f\left(x_{1} t\right)\left[G_{2}(t)-H_{2}(t)\right] d t<H_{1}(0)_{\epsilon} / 2
$$

(Here, we have used the assumption that $G_{2}(0)=N(0)=0$.) Letting $H=$ $H_{1}-H_{2}$, we have

$$
\int_{0}^{1} f\left(x_{1} t\right)[H(t)-G(t)] d t<H_{1}(0) \epsilon=H(0) \epsilon
$$

Defining

$$
P(x)=\int_{0}^{1} f(x t)[H(t)-G(t)] d t
$$

it follows that $P\left(x_{1}\right)<H(0) \epsilon$. Note, as in the proof of Theorem 1 , that $P$ is continuous on $(0,1]$. We have

$$
\int_{0}^{1} f(x t) H(t) d t=P(x)+q(x) f(x)
$$

for almost all $x$ in $(0,1)$, and an integration by parts yields

$$
\int_{0}^{1}[F(x t)-F(x)] H^{\prime}(t) d t=H(0) F(x)-x[P(x)+q(x) f(x)] .
$$

Here, again as in the proof of Theorem $1, q\left(x_{1}\right) f\left(x_{1}\right) \leqq 0$. Hence,

$$
\begin{aligned}
0 & \geqq \int_{0}^{1}\left[F\left(x_{1} t\right)-F\left(x_{1}\right)\right] H_{1}^{\prime}(t) d t \\
& =H(0) F\left(x_{1}\right)-x_{1}\left[P\left(x_{1}\right)+q\left(x_{1}\right) f\left(x_{1}\right)\right]+\int_{0}^{1}\left[F\left(x_{1} t\right)-F\left(x_{1}\right)\right] H_{2}^{\prime}(t) d t \\
& \geqq H(0) F\left(x_{1}\right)-H(0)_{\epsilon}-T \int_{0}^{1} H_{2}^{\prime}(t) d t=H(0) F\left(x_{1}\right)-H(0)_{\epsilon}-T H_{2}(1) \\
& =H(0) F\left(x_{1}\right)-H(0)_{\epsilon}-T G_{2}(1) \geqq H(0) F\left(x_{1}\right)-H(0)_{\epsilon}-T p G(0) .
\end{aligned}
$$

Since $G(0)=H(0) \neq 0$, we have $F\left(x_{1}\right) \leqq \epsilon+p T$. Since $\epsilon$ was otherwise arbitrary, $F\left(x_{1}\right) \leqq p T<T / 2$, the last strict inequality following from the 
assumption that $f$ is not equivalent to zero on $(0,1)$. Thus, $F\left(x_{1}\right)<T / 2$, contradicting (2.2). This contradiction completes the proof of Theorem 3.

3. The $C^{\infty}$ case.

Theorem 4. Let $\int_{0}^{1} f(x t) G(t) d t=0$ for all $x \in(0,1]$, where

(i) $f \in C^{\infty}[0,1]$,

(ii) $G \in C^{2}[0,1]$ and $G^{\prime}<0$ on $[0,1]$,

(iii) $G(1) \geqq 0$.

Then $f=0$ for all $x \in[0,1]$.

We note that all of the results in $\$ 2$ apply, a fortiori, to the $C^{\infty}$ case. The importance of Theorem 4 lies in the fact that it is not assumed, as in Corollary 1 , that $G^{\prime}$ is nonincreasing. For the proof of Theorem 4, we have

Lemma 2. Let $g \in C^{\prime}[0,1]$ with $g(x)>0$ on the closed interval $[0,1]$. Then there exists a positive integer $n$ such that the function $\bar{g}(x)=x^{n} g(x)$ is nondecreasing on $[0,1]$.

Proof. Let $2 K=\min \{g(x) \mid 0 \leqq x \leqq 1\}$ and $L=\max \left\{\left|g^{\prime}(x)\right| \mid 0 \leqq x \leqq 1\right\}$. Then $K>0$ and, by the Stone-Weierstrass Theorem, there exists a sequence $\left\{P_{m}(x)\right\}$ of polynomials such that

(i) $P_{m}(x) \rightarrow g(x)$ uniformly on $[0,1]$,

(ii) $P_{m}(x) \geqq K$ for all $m$ and all $x \in[0,1]$,

(iii) $\left|P_{m}^{\prime}(x)\right| \leqq L+1$ for all $m$ and all $x \in[0,1]$.

Let $n$ be an integer satisfying $n>(L+1) / K$. Then for all $m$ and all $x \in(0,1]$,

$$
\frac{d}{d x} x^{n} P_{m}(x)=\left[n+\frac{x P_{m}^{\prime}(x)}{P_{m}(x)}\right] x^{n-1}>\left[n-\frac{L+1}{K}\right] x^{n-1}>0,
$$

and $x^{n} P_{m}(x)$ is strictly increasing on $[0,1]$. Finally, let $x$ and $y$ be arbitrary numbers in $[0,1]$ with $x<y$ and let $\epsilon$ be an arbitrary positive number. Choosing $m$ so that $\left|g(t)-P_{m}(t)\right|<\epsilon / 2$ for all $t \in[0,1]$, we have

$$
\begin{aligned}
y^{n} g(y)-x^{n} g(x) & =y^{n}\left[g(y)-P_{m}(y)\right]+y^{n} P_{m}(y)-x^{n} P_{m}(x)+x^{n}\left[P_{m}(x)-g(x)\right] \\
& >-\epsilon / 2+0-\epsilon / 2=-\epsilon .
\end{aligned}
$$

Since $\epsilon$ is otherwise arbitrary, $y^{n} g(y)-x^{n} g(x) \geqq 0$, showing that $x^{n} g(x)$ is nondecreasing on $[0,1]$.

Proof of Theorem 4. Let $g(x)=-G^{\prime}(x)$ and $F(x)=\int_{0}^{x} f(t) d t$. Then $g \in C^{\prime}[0,1]$ and $g(x)>0$ on the closed interval $[0,1]$. By the lemma, there exists an integer $n$ such that $x^{n+1} g(x)$ is a nondecreasing function on $[0,1]$. By an integration by parts, 


$$
\int_{0}^{1} F(x t) g(t) d t=-G(1) F(x) \text { for all } x \in[0,1]
$$

Successive differentiation of this equation yields

$$
\int_{0}^{1} f^{(n)}(x t) t^{n+1} g(t) d t=-G(1) f^{(n)}(x), \quad x \in[0,1] .
$$

Since $G(1) \geqq 0$, it follows by Theorem 1 that $f^{(n)}(x) \equiv 0$ for all $x \in[0,1]$. Thus $f$ is a polynomial on $[0,1]$. This, together with the integral equation, implies that $f=0$ for all $x \in[0,1]$.

4. Examples. We present two simple examples which show that stringent conditions, such as those above, must be placed on the function $G$ in order to ensure that $\int_{0}^{1} f(x t) G(t) d t=0$ implies that $f$ is equivalent to zero:

(a) Let $G(t)=24 t^{2}-37 t+15$. ( $G$ is strictly positive on the interval $[0,1]$.) Then there exists a bounded function $f$ on $[0,1]$ which is not equivalent to zero and such that $\int_{0}^{1} f(x t) G(t) d t=0$ for every $x \in[0,1]$. Indeed, a calculation shows that

$$
\int_{0}^{1} t^{z} G(t) d t=0
$$

with $z=1 / 4 \pm i \sqrt{215} / 4$. Thus $\operatorname{Re} \int_{0}^{1}(x t)^{z} G(t) d t \equiv 0$; i.e.,

$$
\int_{0}^{1} f(x t) G(t) d t \equiv 0, \quad \text { with } f(x)=x^{1 / 4} \cos \left(\frac{\sqrt{215}}{4} \ln x\right) .
$$

(b) Let $G_{a}(t)=2$ for $0 \leqq t \leqq 1 / 2$ and $G_{a}(t)=a$ for $1 / 2<t \leqq 1 \quad(0<a<2)$. Here the equation

$$
\int_{0}^{1} f(x t) G_{a}(t) d t=0
$$

is equivalent to

$$
2 F(x / 2)+a[F(x)-F(x / 2)]=0,
$$

with $F(x)=\int_{0}^{x} f(t) d t$. Attempting a solution $F(x)=x^{\beta}$ (with real part of $\beta$ to be greater than zero in order to ensure that $\left.f \in L^{1}(0,1)\right)$, we obtain $2^{\beta}=(a-2) / a$, or

$$
\beta=\frac{1}{\ln 2}\left[\ln \frac{2-a}{a}+i \pi\right] \equiv \beta_{1}+i \beta_{2} .
$$

Note that $\beta_{1}>0$ if $a<1$; hence, there exists a nontrivial solution $f$ of (4.1) if the negative variation of $G_{a}(N=2-a)$ is greater than $1 / 2 G_{a}(0)=1$. This example shows the significance of the conditions on $G$ of Theorem 3 . 
It also shows that the condition $G$ nonincreasing is not enough to ensure that $f$ is equivalent to zero.

Two remarks concerning other applications of the above theorems should be made in connection with these two examples. Firstly, the theorems are applicable to the problem of showing that an analytic function of the form $H(z)=\int_{0}^{1} t^{z} G(t) d t$ has no zeros in the half-plane $\operatorname{Re} z>-1$. This problem occurs, for example, in the Mellin transform solution of integral equations (cf. [1]). Secondly, the theorems pertain to the class of homogeneous functional equations which are generated by equations of the form $\int_{0}^{1} f(x t) G(t) d t$ $=0$, with $G$ a step function on $[0,1]$. An example of this is the functional equation for $F$ in example (b) above.

5. Another application. A variant of the argument used in the proof of Theorem 4 yields

Theorem 5. The incomplete gamma function $P(z)=\int_{0}^{1} t^{z-1} e^{-t} d t$ is zerofree in the right half-plane $\operatorname{Re} z>0$.

Proof. Suppose to the contrary that $P(z)=0$ for some $z$ with $\operatorname{Re} z>0$. Let $f(x)=x^{z-1}$ and $G(t)=e^{-t}$. Then

$$
\int_{0}^{1} f(x t) G(t) d t=0 \text { for all } x \in(0,1) \text {. }
$$

An integration by parts, as in the proof of Theorem 4, gives

$$
\int_{0}^{1} f(x t) t e^{-t} d t=-e^{-1} f(x), \quad x \in(0,1) .
$$

Since the function $t e^{-t}$ is increasing on $[0,1]$ and $f \in L^{1}(0,1)$, it follows by Theorem 1 that $f=0$ almost everywhere in $(0,1)$. This contradiction completes the proof.

In the same way we may prove

Theorem 6. The Prym function $P(M, z)=\int_{0}^{M} t^{z-1} e^{-t} d t$ is zero-free in the half-plane $\operatorname{Re} z>M-1$.

\section{REFERENCES}

1. M. A. Evgrafov, Analytic functions, "Nauka", Moscow, 1965; English transl., Saunders, Philadelphia, Pa., 1966. MR 32 \#5843; 33 \#5849.

2. E. W. Hobson, The theory of functions of a real variable and the theory of Fourier series. Vols. I, II, Cambridge Univ. Press, New York, 1927; reprint, Dover, New York, 1958. MR 19, 1166.

3. H. L. Royden, Real analysis, 2nd ed., Macmillan, New York, 1969. MR $27 \# 1540$. 
4. G. Sansone and J. Gerretsen, Lectures on the theory of functions of a complex variable. Vol. I. Holomorphic functions, Noordhoff, Groningen, 1960. MR 22 \#4819.

5. M. Urabe, Potential forces which yield periodic motions of a fixed period, J. Math. Mech. 10 (1961), 569-578. MR 23 \#A391.

6. G. N. Watson, A treatise on the theory of Bessel functions, Cambridge Univ. Press, New York, 1966.

7. E. T. Whittaker and G. N. Watson, A course of modern analysis. An introduction to the general theory of infinite processes and of analytic functions; with an account of the principal transcendental functions, 4th ed., reprinted, Cambridge Univ. Press, New York, 1962. MR 31 \#2375.

DEPARTMENT OF MATHEMATICS, UNIVERSITY OF MONTANA, MISSOULA, MONTANA 59801 